\title{
Nanonet: Low-temperature-processed tellurium nanowire network for scalable p-type field-effect transistors and a highly sensitive phototransistor array
}

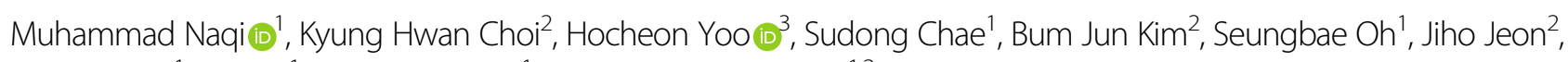
Cong Wang ${ }^{1}$, Na Liu', Sunkook Kim $\mathbb{1}^{1}{ }^{1}$ and Jae-Young Choi $\mathbb{B}^{1,2}$

\begin{abstract}
Low-temperature-processed semiconductors are an emerging need for next-generation scalable electronics, and these semiconductors need to feature large-area fabrication, solution processability, high electrical performance, and wide spectral optical absorption properties. Although various strategies of low-temperature-processed n-type semiconductors have been achieved, the development of high-performance p-type semiconductors at low temperature is still limited. Here, we report a unique low-temperature-processed method to synthesize tellurium nanowire networks (Te-nanonets) over a scalable area for the fabrication of high-performance large-area p-type fieldeffect transistors (FETs) with uniform and stable electrical and optical properties. Maximum mobility of $4.7 \mathrm{~cm}^{2} \mathrm{Ns}$, an on/off current ratio of $1 \times 10^{4}$, and a maximum transconductance of $2.18 \mu \mathrm{S}$ are achieved. To further demonstrate the applicability of the proposed semiconductor, the electrical performance of a Te-nanonet-based transistor array of 42 devices is also measured, revealing stable and uniform results. Finally, to broaden the applicability of p-type Tenanonet-based FETs, optical measurements are demonstrated over a wide spectral range, revealing an exceptionally uniform optical performance.
\end{abstract}

\section{Introduction}

The development of large-area thin-film semiconductors at low temperature $\left(\leq 200^{\circ} \mathrm{C}\right)$ with stable device performance is of great interest for various applications, including flexible electronics, display-panel systems, and logic circuit devices. ${ }^{1-4}$ Specifically, the reported processing techniques for fabricating highperformance devices require a dependable vacuum

\footnotetext{
Correspondence: Sunkook Kim (intel0616@gmail.com) or Jae-Young Choi (jy.choi@skku.edu)

${ }^{1}$ School of Advanced Materials Science and Engineering, Sungkyunkwan University, Suwon, Korea

${ }^{2}$ SKKU Advanced Institute of Nanotechnology (SAINT), Sungkyunkwan University, Suwon, Korea

Full list of author information is available at the end of the article These authors contributed equally: Muhammad Naqi, Kyung Hwan Choi, Hocheon Yoo
}

environment, high-temperature annealing process, and a long processing time; thus, these techniques have limited applicability and are not cost-effective., ${ }^{5,6}$ In this regard, existing low-temperature-processed n-type semiconductor material systems with respectable good device performance, such as amorphous indium gallium zinc oxide (a-IGZO), zinc oxide ( $\mathrm{ZnO})$, indium oxide $\left(\mathrm{In}_{2} \mathrm{O}_{3}\right)$, and cadmium sulfide (CdS), have been investigated; however, these systems have limitations in terms of hightemperature annealing (to improve the crystallinity and stoichiometry), additional doping interactions, and the high cost for scalable additive fabrication. ${ }^{5-8}$ Based on the desire for the low-temperature processing of thin-film semiconductors, there are many studies on various $p$-type semiconductor materials, including organic compounds, metal oxides, and amorphous silicon with extremely low

\section{(-) The Author(s) 2021}

(c) (i) Open Access This article is licensed under a Creative Commons Attribution 4.0 International License, which permits use, sharing, adaptation, distribution and reproduction cc. in any medium or format, as long as you give appropriate credit to the original author(s) and the source, provide a link to the Creative Commons license, and indicate if changes were made. The images or other third party material in this article are included in the article's Creative Commons license, unless indicated otherwise in a credit line to the material. If material is not included in the article's Creative Commons license and your intended use is not permitted by statutory regulation or exceeds the permitted use, you will need to obtain permission directly from the copyright holder. To view a copy of this license, visit http://creativecommons.org/licenses/by/4.0/. 
mobility of $<1 \mathrm{~cm}^{2} / \mathrm{Vs},{ }^{9-11}$ presenting challenges to their wide applicability and requiring an improvement in device performance to reach an acceptable level. On the other hand, among $p$-type materials, polycrystalline germanium (p-Ge) exhibits high carrier mobility $\left(\sim 80 \mathrm{~cm}^{2} / \mathrm{Vs}\right)$ but has limited wide-scale application due to requiring a higher temperature $\left(>400{ }^{\circ} \mathrm{C}\right)$ annealing process and the crystallization of its doped metals. ${ }^{12}$ Additionally, an extensively explored $p$-type semiconductor material, carbon nanotubes (CNTs), exhibits high device performance and has been reported in various applications, including complementary metal-oxide-semiconductor (CMOS) architectures, storage devices, and biosensors, ${ }^{13-17}$ but nanoscale processing over scalable additive fabrication remains a significant concern.

Recently, a promising $p$-type semiconductor material, namely, tellurium, has been increasingly studied in the field of electronic and optoelectronic devices based on its tunable bandgap (an indirect bandgap of approximately $0.35 \mathrm{eV}$ and a direct bandgap of approximately $1.04 \mathrm{eV}$ in bulk and monolayer samples, respectively) and excellent carrier transport properties. ${ }^{18-22}$ Te typically exhibits a trigonal atomic structure in which atoms form helical chains with strong bonding between neighboring atoms based on van der Waals interactions. ${ }^{23-26}$ In previous reports, large-scale polycrystalline Te films have also been prepared and studied using thermal evaporation processes. $^{4,27,28}$ The material properties of Te films are dependent on the deposition temperature and rate, making it difficult to obtain highly crystalline $\mathrm{Te}$ in a scalable area. ${ }^{28,29}$ Alternatively, another approach using tellurium nanowires (TeNWs) has been widely studied, and various methods for synthesizing TeNW films, which differ significantly from bulk Te films, have been investigated. $^{30-34}$ Atomic chains of one-dimensional (1D) Te have been observed in curled shapes over large surface areas, which makes such surfaces very sensitive as fieldeffect transistors (FETs). ${ }^{35-39}$ The stabilization of 1D nanowires has been successfully demonstrated by introducing CNT and boron nitride nanotube (BNNT) encapsulation methods that provide high-performance structural characteristics and active shielding for filling materials, ${ }^{35-39}$ but limits the applicability of this material.

Herein, we report a unique low-temperature-processed $\left(\leq 100^{\circ} \mathrm{C}\right)$ method for the scalable deposition of a tellurium nanowire network (Te-nanonet) to fabricate highperformance FETs with stable electrical and optical properties. The presented Te-nanonet is patterned by a dry etching process based on a conventional photolithography process, and FET devices based on the patterned Te-nanonet are fabricated through a lift-off process. Additionally, a transistor array of 42 devices based on the Te-nanonet is fabricated, revealing strong electrical properties with excellent uniformity and stability. The proposed Te-nanonet-based phototransistor is also analyzed at different wavelengths of visible light $(405 \mathrm{~nm}, 538 \mathrm{~nm}$, and $632 \mathrm{~nm})$, and the optical results reveal a stable $V_{\text {th }}$ shift, reliable photocurrent $\left(I_{\text {photo }}\right)$, excellent photoresponsivity $\left(95.64 \mathrm{AW}^{-1}\right)$, high detectivity $\left(87.34 \times 10^{10}\right)$, linear sensitivity, and desirable rise/fall time responses $(5$ and $7 \mathrm{~s}$, respectively. Additionally, the photoresponse of the proposed transistor array exhibits high stability in terms of photocurrent $\left(I_{\text {photo }}\right)$ and photoresponsivity (R) when exposed to blue light $(405 \mathrm{~nm})$ at a power intensity of $1.2 \mathrm{~mW}$. Thus, this study proposes a unique platform for low-temperature-processed nextgeneration electronic applications.

\section{Materials and methods \\ Synthesis of Te-nanonet}

Te-nanonets were grown using a hydrothermal method in the presence of polyvinylpyrrolidone (PVP) as a capping agent. First, $2 \mathrm{mmol}$ of $\mathrm{Na}_{2} \mathrm{TeO}_{3}$ (99\%, Sigma Aldrich, USA) and $2.4 \mathrm{~g}$ of PVP (average M.W. 58,000, Alfa Aesar, USA) were dissolved in $84 \mathrm{~mL}$ of deionized water to form a clear solution. Next, $4 \mathrm{~mL}$ of hydrazine monohydrate (80\%, DAEJUNG, Korea) and $8 \mathrm{~mL}$ of an aqueous ammonia solution (28\%, JUNSEI, Japan) were added. Then, the solution was sealed in a Teflon-lined stainless steel autoclave. The solution was reacted at $160{ }^{\circ} \mathrm{C}$ for $4 \mathrm{~h}$ and rapidly cooled to room temperature with running water. The products were precipitated by adding $160 \mathrm{~mL}$ of acetone and then washed with deionized water and ethanol repeatedly. Finally, the washed samples were dispersed in $20 \mathrm{~mL}$ of ethanol and centrifuged at $10,000 \mathrm{rpm}$ for $10 \mathrm{~min}$ to remove any unintended Te flakes and obtain a Te-nanonet solution for further experiments.

\section{Material characterization}

Field-emission scanning electron microscopy (SEM) (Hitachi, S-4300SE) was used for the morphological analysis of the Te-nanonet. Powder x-ray diffraction (XRD) (Rigaku, Ultima IV) was performed using a $\mathrm{Cu} \mathrm{K \alpha}$ radiation source $(\lambda=0.154 \mathrm{~nm})$ on a dried Te-nanonet sample to measure diffraction patterns. Atomic force microscopy (AFM) (Park systems, NX10) was used in tapping mode for the topographic analysis of the barcoated Te-nanonet. Raman spectroscopy (Witec, Alpha $300 \mathrm{M}+$ ) was conducted using a $532 \mathrm{~nm}$ excitation laser to measure the Raman phonon modes of the Te-nanonet. Ultraviolet-visible (UV-vis) spectrophotometry (Agilent, Cary5000) was used to measure the UV absorption of the Te-nanonet solution. A spectrophotofluorometer (Horiba Scientific, Fluorolog3 with TCSPC) was employed to measure the photoluminescence (PL) spectrum of the Tenanonet. Thermogravimetric and differential scanning calorimetry (TG-DSC) (TA Instruments, SDT Q600) 
measurements were performed in air and $\mathrm{N}_{2}$ atmospheres at a heating rate of $20{ }^{\circ} \mathrm{C}$ min-1 for thermal stability analysis. The concentration of the Te-nanonet solution and substrate loading level was confirmed by inductively coupled plasma-optical emission spectroscopy (ICP-OES) (Agilent 7500, Agilent Technologies Inc.).

\section{Preparation of the Te-nanonet thin film}

Te-nanonets were uniformly and densely coated onto $300 \mathrm{~nm} \mathrm{SiO} / / \mathrm{Si}$ substrates using a bar-coating method with a doctor blade. Prior to coating, the substrates were treated with UV/ozone and 3-aminopropyltrimethoxysilane to remove any contaminants from their surfaces and functionalize their surfaces to enhance coating efficiency. Then, the Te-nanonet solution was dropped onto the $1.5 \times 1.5 \mathrm{~cm}$ substrates and bar coated three to five times, forming homogeneous Te-nanonet thin films across the entire surfaces. Finally, the Te-nanonet-coated substrates were dried in a vacuum oven at $50{ }^{\circ} \mathrm{C}$ prior to their use for device fabrication.

\section{Fabrication of the Te-nanonet-based phototransistor}

After coating the Te-nanonet onto $\mathrm{Si}$ wafer substrates, the Te-nanonet was patterned using a dry etching process. To pattern the Te-nanonet, photoresist (PR) (AZGXR601, MERCK, USA) was spin-coated onto the surface of the Te-nanonet at $3000 \mathrm{rpm}$ for $20 \mathrm{~s}$, exposed to UV light for $1 \mathrm{~s}$ with a patterned mask, and then developed in a developer solution (AZ-300MIF, MERCK, USA) for $20 \mathrm{~s}$. After developing the patterns, the devices were annealed at $80{ }^{\circ} \mathrm{C}$ for $30 \mathrm{~min}$, and the unwanted Te-nanonet was etched using $\mathrm{O}_{2}$ plasma treatment (reactive ion etching system) for $60 \mathrm{~s}$ at a power of $60 \mathrm{~W}$ and flow rate of $50 \mathrm{sccm}$. After cleaning the samples with acetone, the source and drain electrodes were patterned using a liftoff process in which the lift-off resist (LOR) (LOR3B, Product \# G3167070500L1GL, MicroChem, USA) was spin-coated for $45 \mathrm{~s}$ at $2000 \mathrm{rpm}$ and annealed at $100^{\circ} \mathrm{C}$ for $20 \mathrm{~min}$. The regular PR was then spin-coated for $20 \mathrm{~s}$ at $3000 \mathrm{rpm}$ and annealed at $90^{\circ} \mathrm{C}$ for $90 \mathrm{~s}$. Next, the substrates were exposed to UV light for $1 \mathrm{~s}$ with a patterned mask and developed in a developer solution for $20 \mathrm{~s}$. Metals ( $\mathrm{Ti} / \mathrm{Au}$ ) with thicknesses of 20 and $100 \mathrm{~nm}$ were then deposited using an e-beam evaporator, and the unwanted metal areas were removed using a remover solution ( $\mathrm{mr}$-Rem 700, Micro-Resist Technology, Germany). The samples were then cleaned in deionized water. After drying the samples, an encapsulation layer of $\mathrm{Al}_{2} \mathrm{O}_{3}(20 \mathrm{~nm})$ was deposited using the atomic layer deposition (ALD) method at $100{ }^{\circ} \mathrm{C}$. Finally, the source and drain were patterned using a simple wet etching process in which PR was spin-coated at $3000 \mathrm{rpm}$ for $20 \mathrm{~s}$, followed by development in a developer solution for $20 \mathrm{~s}$, which in turn was followed by exposure to UV light under a patterned mask.
After patterning the source and drain, the samples were etched in a diluted buffer oxide etchant for $30 \mathrm{~s}$.

\section{Electrical and optical characterizations}

Electrical characteristics were measured using a semiconductor characterization system (Keithley, 4200 SCS). Optical characteristics were measured under visible light illumination at wavelengths of $405 \mathrm{~nm}, 638 \mathrm{~nm}$ (Thorlabs, S405-HP for $405 \mathrm{~nm}$ and SM600 for $638 \mathrm{~nm}$ ), and $532 \mathrm{~nm}$ (Changchun New Industries Optoelectronics Technology Co., MGL-FN-532) at room temperature.

\section{Results and discussion}

\section{Synthesis and characterization of the Te-nanonet}

Te represents a true 1D system composed of triangular helical Te atomic chains in a hexagonal array along the caxis. In this structure, atomic chains are connected by weak van der Waals interactions, and each Te atom in an atomic chain is only covalently bonded with its two neighboring atoms, resulting in equilateral triangle shapes in projections along the longitudinal direction (Fig. 1a). ${ }^{23-26}$ In this study, the Te-nanonet was synthesized using a hydrothermal method through the reduction of sodium tellurite with hydrazine hydrate in the presence of PVP as a capping agent (see the Materials and methods section). ${ }^{31}$ The morphology of the products is strongly dependent on the reaction parameters, including the temperature and presence of proper capping agents, leading to the formation of either nanowires or nanorods (Fig. S1a and b). To ensure a uniform network structure in the deposition step, it is necessary to prepare a high aspect ratio of the nanowire with a constant diameter in the synthesis process. Tenanonets grown at a solution temperature of $160{ }^{\circ} \mathrm{C}$ with PVP are several hundred microns in length with diameters ranging from 10 to $15 \mathrm{~nm}\left(\sim 10^{4}\right.$ aspect ratio), as shown in Fig. 1b. The Raman spectrum of the Te-nanonet reveals blueshifts relative to that of bulk Te for three vibration modes $\left(E_{1}, A_{1}\right.$, and $\left.E_{2}\right)$, which correspond to bond bending, chain expansion (where each atom moves in the basal plane), and bond stretching, respectively. Additionally, the intensities of the $E_{1}$ and $E_{2}$ modes are weaker in the Tenanonet (Fig. 1c). These results are in good agreement with previous reports, which have indicated shifting toward higher frequencies with a decreasing number of Te atomic chains based on the weakening of interchain van der Waals interactions and the enhancement of intrachain covalent interactions. $^{21,35,40}$ The XRD peaks of the obtained Tenanonet match well with the calculated reference and are free of any impurities (Fig. 1d). Both the XRD and Raman results indicate the successful preparation of Te-nanonets. A dispersion of the Te-nanonet in ethanol with a deep blue color (inset in Fig. 1e) was analyzed using a UV-vis spectrometer and PL spectrophotometer to characterize its optical properties. The UV-vis absorption spectrum 


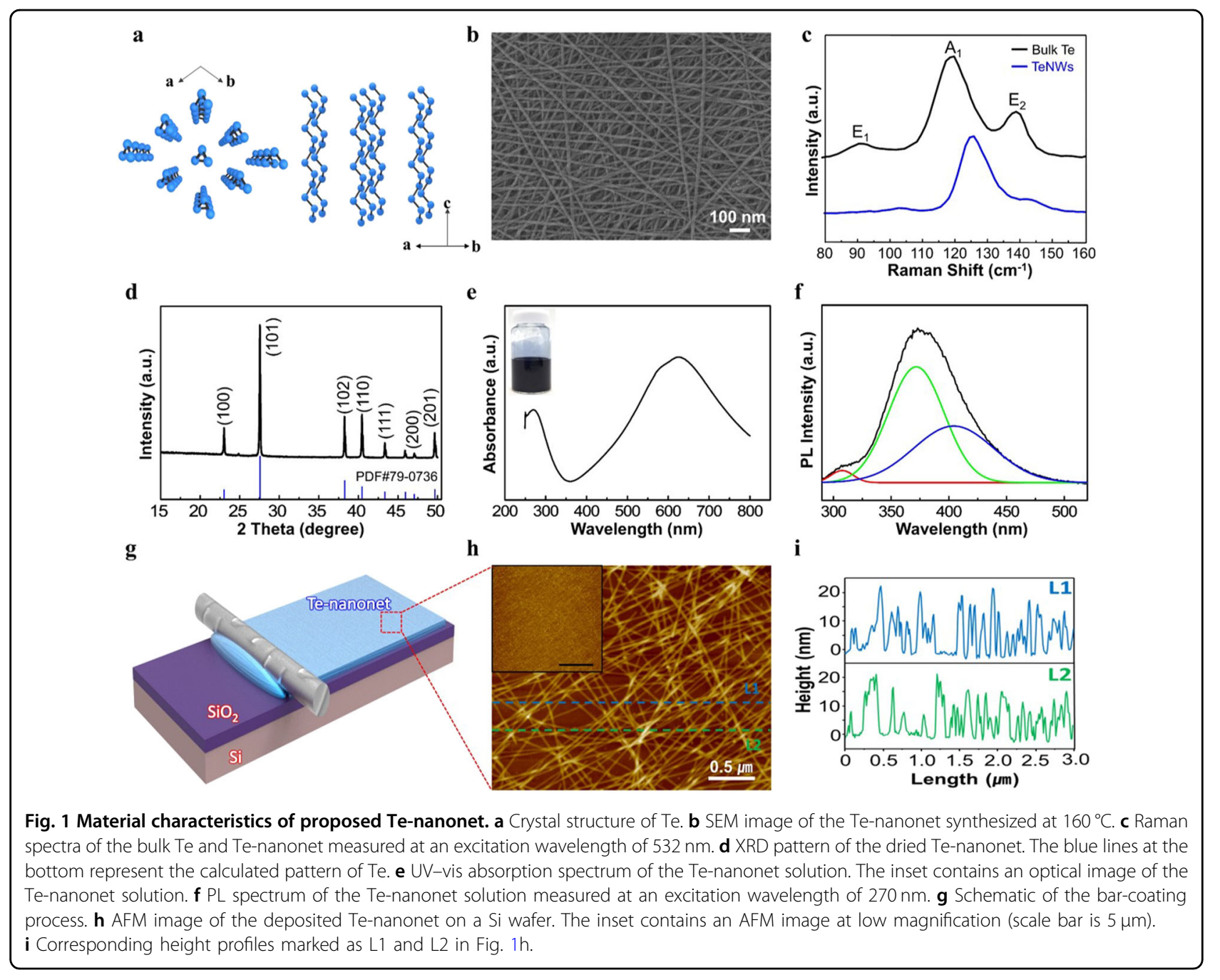

exhibits two broad absorption peaks at 270 and $625 \mathrm{~nm}$ (Fig. 1e). The absorption peak at $270 \mathrm{~nm}$ is caused by a transition from the valence band ( $p$-bonding triplet) to the conduction band ( $p$-antibonding triplet), and the peak at $625 \mathrm{~nm}$ is attributed to a forbidden direct transition. ${ }^{31,41,42}$ The PL spectrum of the Te-nanonet exhibits a broad emission band over the range of $300-500 \mathrm{~nm}$ at an excitation wavelength of $270 \mathrm{~nm}$ (Fig. 1f). The deconvolution of this band yields three Gaussian peaks centered at 307, 371 , and $404 \mathrm{~nm}$. This emission band can be attributed to the absorption peak at $270 \mathrm{~nm}$, implying that the synthesized Te-nanonet can emit in the blue-violet region. To confirm the thermal stability of the Te-nanonet, TG-DSC measurements were conducted in air and $\mathrm{N}_{2}$ atmospheres. Regarding the results in air, there is an upturn in the mass percentage at temperatures above $170^{\circ} \mathrm{C}$, indicating the oxidation of the Te-nanonet and the formation of other oxide phases, whereas the mass percentage is relatively stable at temperatures up to $300{ }^{\circ} \mathrm{C}$ in $\mathrm{N}_{2}$ (Fig. S2a and b). Additionally, it is confirmed that the Te-nanonet on $\mathrm{SiO}_{2}$ substrates completely decomposes and disappears when annealed at $200^{\circ} \mathrm{C}$ under vacuum conditions (Fig. S2c). These results demonstrate that device fabrication at low temperatures is required for Te-nanonet-based devices. The large-area deposition of the Te-nanonet for device fabrication was conducted using a bar-coating method with a doctor blade and a dispersion of the Te-nanonet in ethanol (Figs. 1g and S3a). To form a stable network structure during the deposition process, it is essential to select a solvent capable of dispersing the Te nanowires at a high concentration. If the concentration of nanowire solution is low, it is difficult to achieve a loading level sufficient to form a network structure. The Te nanowires synthesized by the PVP-assisted hydrothermal method in this study have excellent dispersibility in ethanol or water. In particular, it is confirmed by ICP-OES that the Te nanowire solution in ethanol has a high concentration of up to $0.72 \mathrm{mg} \mathrm{mL}^{-1}$ even after centrifugation at $10,000 \mathrm{rpm}$ to remove some unintended Te flakes in the final synthesis step. Based on this result, ethanol was chosen as the 
solvent in consideration of its high dispersion concentration as well as the ease of solvent evaporation during the coating process. The concentrated Te-nanonet solution was dropped onto a $1.5 \times 1.5 \mathrm{~cm}^{2} \mathrm{Si}$ wafer and uniformly coated using the doctor blade at a fixed spacing of $710 \mu \mathrm{m}$. The overall coating process of Te-nanonet in a visual form is also elaborated in Supplementary Video S1. After coating, AFM measurements confirm that the Te-nanonet is deposited onto the entire $\mathrm{Si}$ wafer with a thickness of approximately $20 \mathrm{~nm}$ (Fig. 1h, i), achieving a loading level of $6.94 \mathrm{\mu g} \mathrm{cm}^{-2}$ on the substrate.

\section{Fabrication and electrical characteristics of the Te- nanonet-based transistor}

Phototransistors based on nanowires have not been extensively studied to obtain good electrical and optical properties for scalable processing. Herein, we propose a combination of dry etching and lift-off processes to fabricate scalable phototransistors based on a uniformly dispersed Te-nanonet. First, a solution-type Te-nanonet was deposited onto a $\mathrm{Si}$ wafer using a bar-coating process, as discussed above. To ensure smoothness and uniformity, the bar-coated Te-nanonet was annealed at $80{ }^{\circ} \mathrm{C}$ for $1 \mathrm{~h}$, followed by a dry etching process for patterning the Te-nanonet using a PR coating. The dry etching process was performed using $\mathrm{O}_{2}$ plasma treatment at
$50 \mathrm{sccm}$ and with a power of $60 \mathrm{~W}$ to remove unwanted areas of the Te-nanonet, followed by cleaning with acetone to remove any remaining residues of PR. Clear pattering of the Te-nanonet on the Si wafer is confirmed through AFM measurements, which reveal a thickness of approximately $20 \mathrm{~nm}$, as shown in Fig. S3b. The lift-off process was used to pattern the source and drain electrodes with LOR and PR coatings, respectively. Then, the electron beam (e-beam) method was used to deposit Ti/ $\mathrm{Au}$ with thicknesses of $20 / 100 \mathrm{~nm}$, respectively. The unwanted electrode area was then removed using a chemical remover (mr-Rem 700) and dried for $30 \mathrm{~min}$. The ALD method was used to deposit an encapsulated layer of $\mathrm{Al}_{2} \mathrm{O}_{3}$ with a thickness of $10 \mathrm{~nm}$. To contact the electrodes for electrical measurements, the source and drain electrodes were patterned using a wet etching process consisting of a lithography process and cleaned with acetone. The sequential fabrication process for the proposed Te-nanonet-based phototransistor is presented schematically and photographically in Fig. 2a and b, respectively. Following the fabrication of the Te-nanonetbased phototransistor, its transistor characteristics were measured. The transfer $\left(I_{\mathrm{D}}-V_{\mathrm{G}}\right)$ curve exhibits clear $p$ type behavior with an on/off ratio on the order of $1 \times 10^{4}$ at a $V_{\mathrm{D}}$ of $-10 \mathrm{~V}$ and a threshold voltage of $32.5 \mathrm{~V}$, as shown in Fig. 2c. Additionally, the effective mobility is
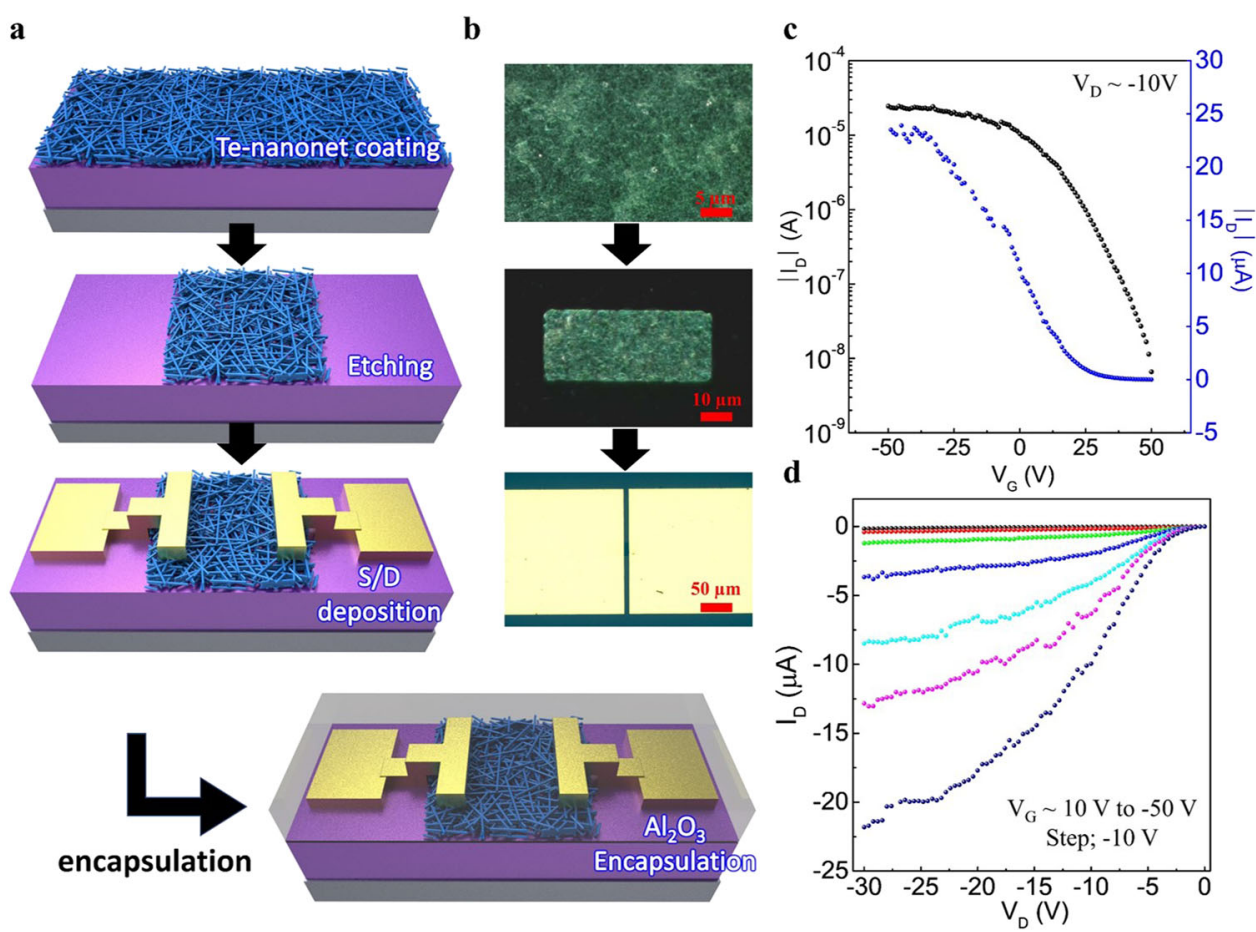

Fig. 2 Fabrication steps and electrical measurements of proposed Te-nanonet transistor. $\mathbf{a}$, $\mathbf{b}$ Sequential fabrication process of the Te-nanonet-based transistor in schematic and real-image layouts, respectively. c Transfer curve $\left(I_{D}-V_{G}\right)$ of a single Te-nanonet-based transistor at $V_{D}=-10 \mathrm{~V}$. $\mathbf{d}$ Output curve $\left(I_{D}-V_{D}\right)$ of the same device at different gate voltages ranging from $10 \mathrm{~V}$ to $-50 \mathrm{~V}$ with a step of $-10 \mathrm{~V}$. 
calculated as $\mu_{\text {eff }}=L g_{m} / W C_{o x} V_{D}$, resulting in a maximum value of $4.7 \mathrm{~cm}^{2} / \mathrm{Vs}$ with a channel length and width of 7 and $20 \mu \mathrm{m}$, respectively. Linear behavior at high $V_{D}$ levels and high current saturation at low $V_{D}$ levels can be observed in the output curve with an applied gate-bias voltage in the range of $10 \mathrm{~V}<V_{\mathrm{G}}<-50 \mathrm{~V}$ and an interval of $-10 \mathrm{~V}$, as shown in Fig. 2d. As a result, the electrical characteristics, specifically, the output curve, depict pronounced linear behavior at high levels of $V_{\mathrm{D}}$, realizing ohmic Te-nanonet-electrode contacts. ${ }^{27,43}$

\section{Scalable electrical performance of the Te-nanonet transistors}

To analyze the uniformity of the electrical characteristics of the proposed Te-nanonet transistors, a transistor array containing 42 devices was fabricated (Fig. 3a). The transfer curve of this transistor array exhibits $100 \%$ yield and high uniformity, demonstrating clear $p$-type behavior (Fig. 3b). Additionally, the transfer curve (linear scale) of the transistor array is presented in the inset in Fig. 3b. To analyze the proposed Te-nanonet-based transistor array characteristics further, the transistor properties were examined in terms of the threshold voltage $\left(\mathrm{V}_{\mathrm{th}}\right), I_{\mathrm{ON}} / I_{\mathrm{OFF}}$ ratio, mobility $(\mu)$, and transconductance $\left(\mathrm{g}_{\mathrm{m}}\right)$, as shown in Fig. 3c-f. The threshold voltage of the Te-nanonet transistors lies in the range of $31-40 \mathrm{~V}$, as shown in Fig. 3c. The transistors exhibit a highly stable on/off ratio on the order of approximately $10^{3}-10^{4}$, as shown in Fig. 3d. A uniform mobility $(\mu)$ is achieved for all transistors in the range of $2.3-4.7 \mathrm{~cm}^{2} / \mathrm{Vs}$, as shown in Fig. 3e. The maximum transconductance $\left(\mathrm{g}_{\mathrm{m}}\right)$ value of the transistor array is also calculated in a small range of approximately $2.18-0.71 \mu \mathrm{S}$, as shown in Fig. 3f. These results indicate that the proposed Te-nanonet-based transistor array exhibits highly uniform transistor characteristics under ambient conditions, thereby providing an advanced platform for future electronic and optoelectronic applications.

\section{Optical measurements of the scalable Te-nanonet-based phototransistors}

The optical properties of the proposed Te-nanonet-based phototransistor were analyzed at three different wavelengths of visible light $(405 \mathrm{~nm}, 538 \mathrm{~nm}$, and $632 \mathrm{~nm}$, power intensity of $1.2 \mathrm{~mW}$ ) and in the dark at room temperature, as indicated by the 3D section view in Fig. 4a. Additionally, a gradual increase in current was applied relative to the dark conditions (Fig. 4b). Exposure to blue light (405 nm) yields a higher photogeneration current than exposure to green $(538 \mathrm{~nm})$ or red $(632 \mathrm{~nm})$ light, owing to the greater photon energy of blue light $(405 \mathrm{~nm}) .{ }^{44}$ The photoresponsivity $(R)$ and detectivity $\left(D^{*}\right)$ of the proposed Tenanonet-based phototransistor were also calculated as key figures of merit for photodetection, as shown in Fig. 4c, d, respectively. Photoresponsivity represents a measurement of photodetection gain, which can be calculated as $R=I_{p h} /$ $P_{\text {inc }}\left(\mathrm{AW}^{-1}\right)$, where $I_{p h}$ denotes the photocurrent and $P_{\text {inc }}$ denotes the incident light power. Here, the photocurrent is calculated as $I_{p h}=I_{\text {total }}-I_{\text {dark }}$, where $I_{\text {total }}$ denotes the total measured current at a specific incident power, and $I_{\text {dark }}$ denotes the current obtained in the dark. Photodetectivity is calculated as $D^{\prime \prime}=R A^{1 / 2}(2 \text { eldark })^{1 / 2}$, where $R$ denotes the photoresponsivity, $A$ denotes the illuminated area, $e$ denotes the elementary charge, and $I_{\text {dark }}$ denotes the measured current in the dark. The responsivity of the Te-nanonetbased phototransistor exhibits linear behavior and clear distinctions between different light illumination wavelengths, as shown in Fig. 4c. The maximum responsivity can be observed with clear linear trends in the range of approximately $95.64-60.09 \mathrm{AW}^{-1}$ at wavelengths ranging from 405 to $638 \mathrm{~nm}$ at the same power of $1.2 \mathrm{~mW}$. High detectivity in the range of approximately 87.33-46.43 $\left(\times 10^{10}\right.$ Jones) is measured at different wavelengths ranging from 405 to $638 \mathrm{~nm}$ at the same power of $1.2 \mathrm{~mW}$, indicating highly linear photodetection properties (Fig. 4d). To verify the photodetection uniformity and stability of the Tenanonet-based transistor array, all 42 devices were examined under blue light $(405 \mathrm{~nm})$ illumination at the same power intensity of $1.2 \mathrm{~mW}$ to calculate their photodetection abilities, namely, the photocurrent $\left(I_{p h}\right)$ and responsivity $(R)$, which are presented in Fig. 4e, f, respectively. The measured photocurrent $\left(I_{p h}\right)$ values of all transistors exhibit high stability and uniformity in the range of approximately $4 \times 10^{-6}$ to $2 \times 10^{-5} \mathrm{~A}$, as shown in Fig. 4e. The responsivity $(R)$ of the proposed Te-nanonet-based transistor array exhibits a uniform response in terms of light detection in the range of approximately $50-90 \mathrm{AW}^{-1}$, as shown in Fig. 4f. These results demonstrate that the proposed Te-nanonet-based phototransistor exhibits excellent scalable photoresponsive performance with high uniformity and stability.

\section{Photodetection key figure analysis of the Te-nanonet- based phototransistor}

To further evaluate the photoresponsive behavior of the Te-nanonet-based transistors, several key figures of merit for photodetection, namely, the threshold voltage difference $\left(\Delta V_{t h}\right)$, photocurrent $\left(I_{p h}\right)$, responsivity $(R)$, specific detectivity $(* D)$, sensitivity $(S)$, and time-domain behavior, were analyzed. The threshold voltage difference $\left(\Delta V_{t h}\right)$ is calculated as $\Delta V_{t h}=V_{\text {th_light }}-V_{\text {th_dark, }}$, where $V_{t h \_l i g h t}$ denotes the threshold voltage at a specific light power intensity, and $V_{t h \_ \text {dark }}$ denotes the threshold voltage in the dark. Photosensitivity $(S)$ is calculated as $S=I_{\mathrm{ph}} / I_{\text {dark }}$, where $I_{p h}$ denotes the photocurrent and $I_{\text {dark }}$ denotes the current in the dark. Figure $5 \mathrm{a}$ presents the variations in the threshold voltage difference $\left(\Delta V_{t h}\right)$ of the Te-nanonetbased phototransistors at different wavelengths of $405 \mathrm{~nm}$, $538 \mathrm{~nm}$, and $632 \mathrm{~nm}$ with various power intensities in the range of $0.2-1.2 \mathrm{~mW}$. The results reveal that $\Delta V_{t h}$ varies 


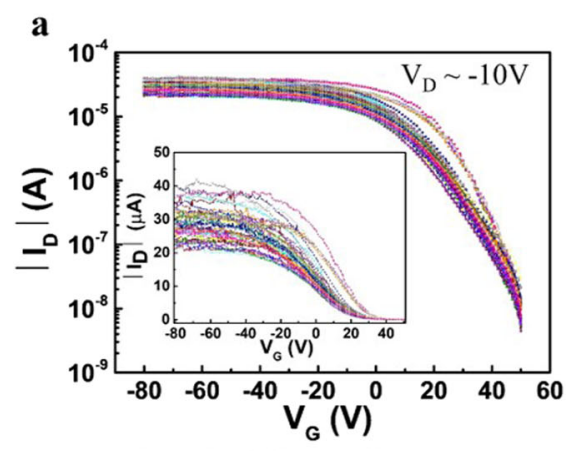

b

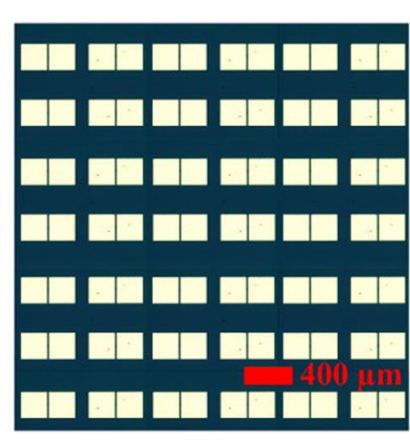

c

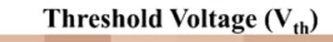

d

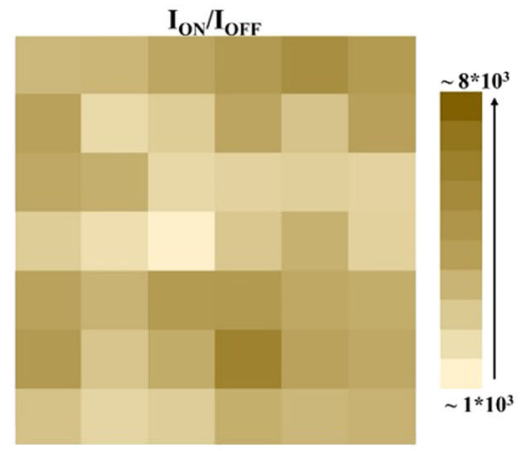

e
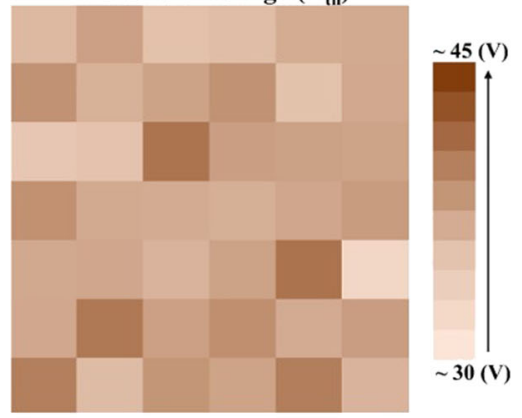

f
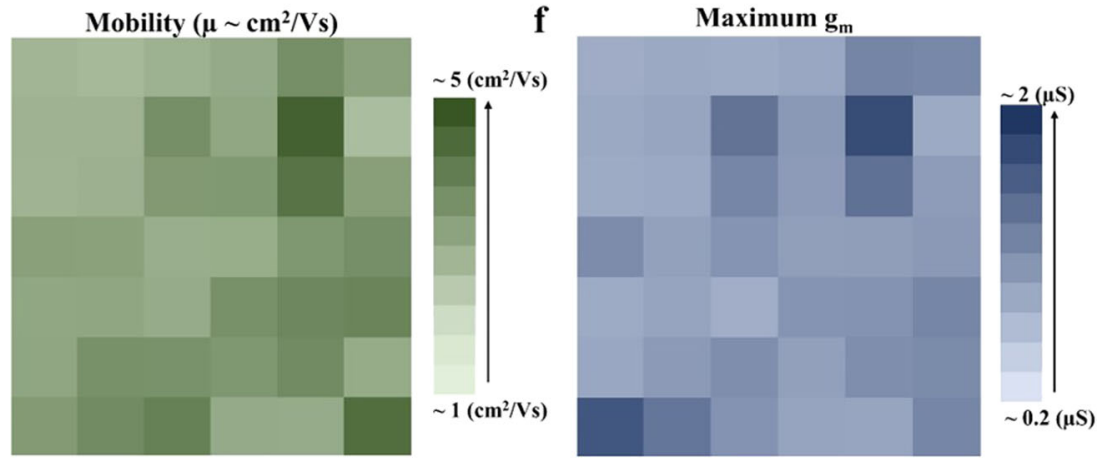

Fig. 3 Electrical properties of proposed Te-nanonet-based transistor array. a Transfer curves $\left(l_{D}-V_{G}\right)$ of 42 devices at $V_{D}=-10 \mathrm{~V}$ on a log scale and linear scale (inset). $\mathbf{b}$ Optical image of the Te-nanonet-based transistor array. c-f Mapping of the performance of 42 devices in terms of the threshold voltage $\left(V_{\text {th }}\right)$, I ON $/ I_{\text {OFF }}$ ratio, mobility $(\mu)$, and maximum transconductance $\left(g_{m}\right)$.

in the ranges of $4.4-25.8 \mathrm{~V}, 3.9-19.7 \mathrm{~V}$, and $3.3-17.6 \mathrm{~V}$ at $405 \mathrm{~nm}, 538 \mathrm{~nm}$, and $632 \mathrm{~nm}$, respectively, when at different power intensities, indicating the high linearity and clear distinction of photodetection. The photocurrent $\left(I_{p h}\right)$ variations of the proposed Te-nanonet-based phototransistor exhibit excellent linear behavior at different power intensities of visible light illumination. The photocurrent and light power are fitted by a functional relationship based on the simple power law, namely, $I_{p h} \sim P_{i n c}{ }^{\alpha}$, where $P_{i n c}$ defines the incident power and $\alpha$ defines the fitting parameter. After fitting the simple power-law equation to the experimental data, the fitting parameter $(\alpha)$ is determined to be $0.91,0.63$, and 0.83 at $405 \mathrm{~nm}, 538 \mathrm{~nm}$, and $632 \mathrm{~nm}$, respectively, thus demonstrating the clear linear distinction of the device (Fig. 5b). Figure 5c presents the responsivity of the device in the ranges of $95.64-78.86 \mathrm{AW}^{-1}$,
73.04-36.14 $\mathrm{AW}^{-1}$, and $60.09-15.06 \mathrm{AW}^{-1}$ at visible spectral wavelengths of $405 \mathrm{~nm}, 538 \mathrm{~nm}$, and $632 \mathrm{~nm}$, respectively, when at different power intensities $(0.2-1.2 \mathrm{~mW})$. Additionally, the responsivity and incident light power $\left(P_{\text {inc }}\right)$ exhibit a power relationship of $\mathrm{R} \sim P_{\text {inc }}^{\beta-1}$, where $\beta$ denotes the fitting parameter. After fitting the experimental data based on power-law behavior, the fitting parameter $(\beta)$ is determined to be $-1.50,-0.40$, and -0.62 at $405 \mathrm{~nm}$, $538 \mathrm{~nm}$, and $632 \mathrm{~nm}$, respectively, thus demonstrating excellent photodetection responses. The specific detectivity of the proposed Te-nanonet-based phototransistor was also measured at the aforementioned wavelengths while at different power intensities ranging from 0.2 to $1.2 \mathrm{~mW}$, as shown in Fig. 5d. The results contain detectivity variations in the ranges of 87.34-72.01, 50.43-24.9, and 46.43-13.23 $\left(" 10^{10}\right.$ Jones) at the different visible light wavelengths of 

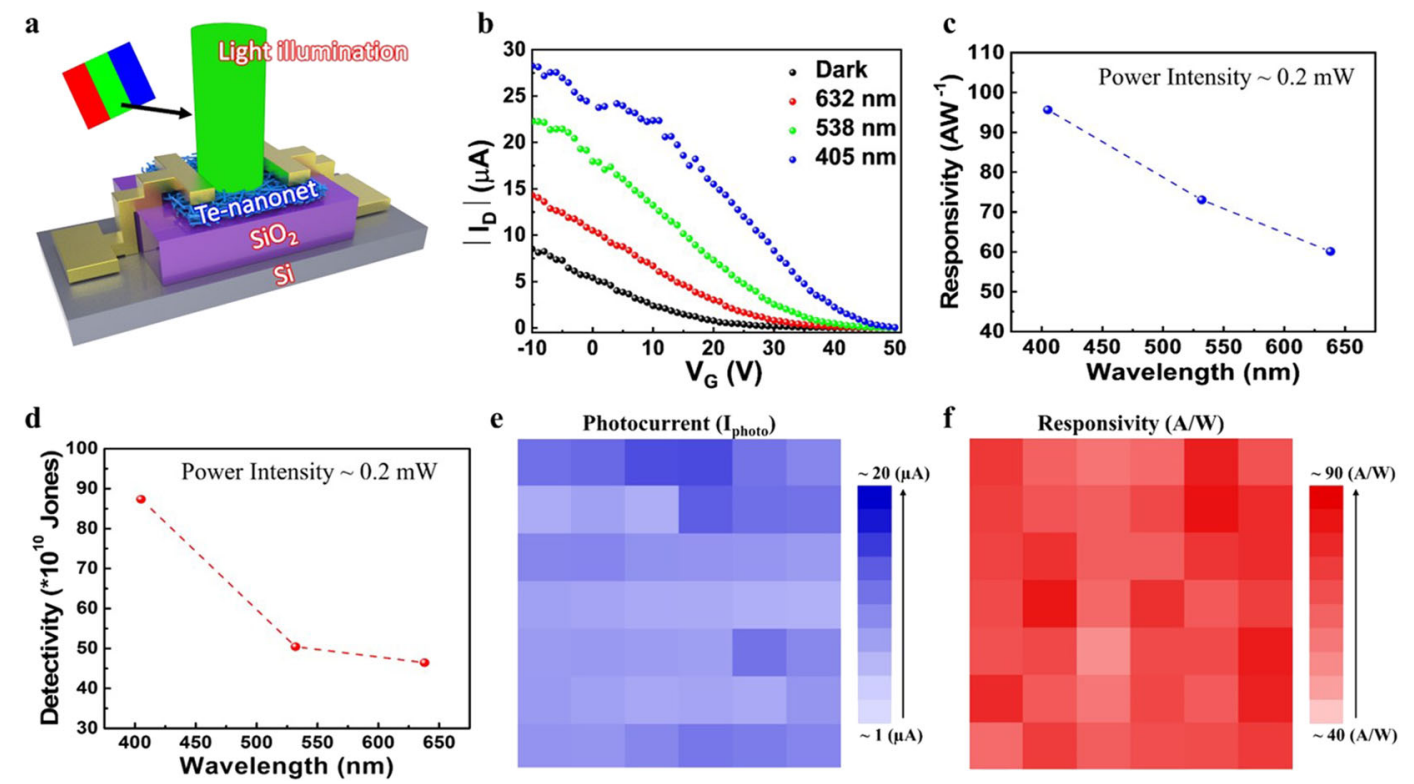

Fig. 4 Optical measurements of proposed Te-nanonet-based phototransistor. a Schematic layout of the proposed Te-nanonet-based transistor under visible light illumination (red, green, and blue). $\mathbf{b}$ Transfer curve $\left(I_{\mathrm{D}}-V_{\mathrm{G}}\right)$ of the proposed device illuminated at different wavelengths of visible light $(405 \mathrm{~nm}, 538 \mathrm{~nm}, 632 \mathrm{~nm})$ and at a power intensity of $1.2 \mathrm{~mW}$ on a linear scale. $\mathbf{c}$, $\mathbf{d}$ Photoresponsivity and specific detectivity of the device at different wavelengths $(405 \mathrm{~nm}, 538 \mathrm{~nm}, 632 \mathrm{~nm}$ ), respectively. e, f Mappings of the 42 devices in terms of the photocurrent and responsivity, respectively, under blue light $(405 \mathrm{~nm})$ illumination at a power of $1.2 \mathrm{~mW}$.

a

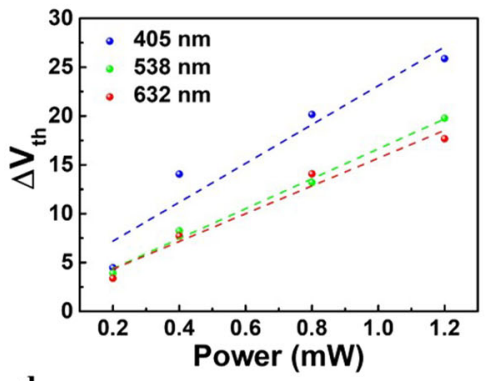

d

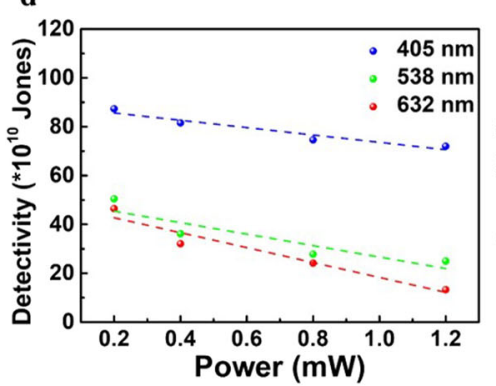

b

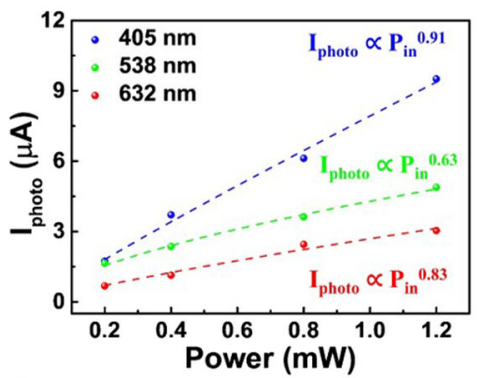

e

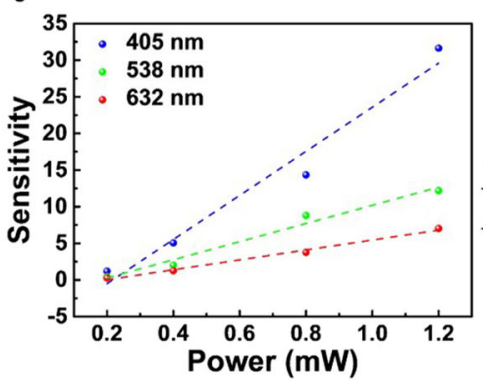

c

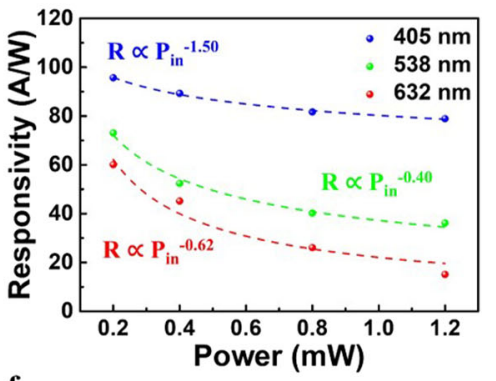

f

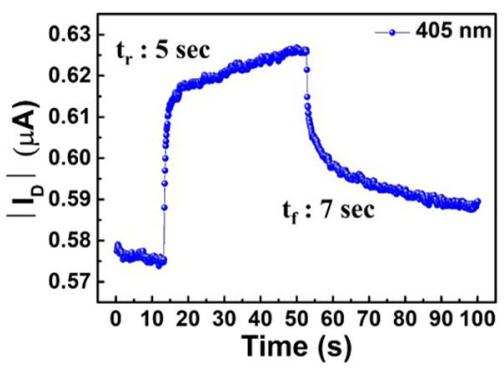

Fig. 5 Photodetection properties of proposed Te-nanonet based phototransistor. a-e Photodetection measurements of the proposed device in terms of the threshold voltage difference $\left(\Delta V_{t h}\right)$, photocurrent $\left(I_{\text {photo }}\right)$, responsivity $(R)$, detectivity $\left({ }^{*} 10^{10}\right.$ Jones), and sensitivity when illuminated at different wavelengths of visible light $(405 \mathrm{~nm}, 538 \mathrm{~nm}$, and $632 \mathrm{~nm}$ ) and at various power intensities ranging from 0.2 to $1.2 \mathrm{~mW}$. f Time-domain measurements of the Te-nanonet-based phototransistor under blue light $(405 \mathrm{~nm})$ illumination at a power intensity of $1.2 \mathrm{~mW}$. 
$405 \mathrm{~nm}, 538 \mathrm{~nm}$, and $632 \mathrm{~nm}$, respectively. In addition to the key figures of merit for photodetection, sensitivity was also measured. Distinct linearity can be observed in the ranges of 1.17-31.62 (405 nm), 0.44-12.16 $(538 \mathrm{~nm})$, and $0.27-7.02(632 \mathrm{~nm})$, as shown in Fig. 5e. In addition, to understand the advantages of the proposed Te-nanonetbased phototransistor, an electrical and optical comparison of the proposed phototransistor with previously reported phototransistors based on nanowires is provided in Table S1, which is broken down by synthesis method, device scaling, material type, mobility, photodetection range, responsivity, and detectivity. Then, the time response behavior of the device was characterized under pulses of visible light at a wavelength of $405 \mathrm{~nm}$ and a power intensity of $0.2 \mathrm{~mW}$. A gradual increase in current with a rise time of $5 \mathrm{~s}$ and fall time of $7 \mathrm{~s}$ can be observed in Fig. 5f. The time-domain behavior of the proposed Te-nanonet-based phototransistor is stable throughout the measurement process, demonstrating the robustness of our photodevice. Although the present contribution is initially intended to obtain the low-temperature uniform synthesis of Te-nanonet on a scalable level with high electrical and optical properties, it can be further enhanced for utilization not only in high-performance rigid devices such as imaging sensors, touch panels, display systems, and large-scale photodetectors but also in a flexible platform for next-generation electronic and optoelectronic devices.

\section{Conclusions}

In conclusion, we introduced a low-temperature processing method to fabricate a highly uniform and stable scalable phototransistor array using a Te-nanonet semiconductor material. A Te-nanonet solution was synthesized using the hydrothermal method and dispersed uniformly on a scalable Si wafer using the bar-coating method. A phototransistor array was fabricated using simple processing techniques at a consistently low temperature $\left(100^{\circ} \mathrm{C}\right)$. A maximum mobility of $4.7 \mathrm{~cm}^{2} / \mathrm{Vs}$ and $\mathrm{I}_{\text {on }} / \mathrm{I}_{\text {off }}$ ratio of $1 \times 10^{4}$ were obtained, and 42 transistor devices were used to determine the stability and uniformity of device performance. The optoelectronic performance of the proposed device was characterized at different wavelengths of visible light $(405 \mathrm{~nm}, 538 \mathrm{~nm}$, and $632 \mathrm{~nm}$ ) and at various power levels ranging from 0.2 to $1.2 \mathrm{~mW}$. Additionally, the photodetection of the Tenanonet-based transistor array was characterized in terms of photocurrent $\left(I_{\text {photo }}\right)$ and responsivity when exposed to visible light at a wavelength of $405 \mathrm{~nm}$ and a power level of $1.2 \mathrm{~mW}$. The results revealed that the proposed device was capable of linear variations in its threshold voltage difference $\left(\Delta V_{t h}\right)$ and photocurrent $\left(I_{\text {photo }}\right)$ and exhibited photosensitivity with high responsivity $\left(95.64 \mathrm{AW}^{-1}\right)$ and excellent detectivity $\left(87.34 \times 10^{10}\right.$
Jones). In this regard, the proposed scalable Te nanonet can potentially be used in next-generation nanoelectronic and optoelectronic applications.

\section{Acknowledgements \\ This research was supported in part by the National Research Foundation of Korea (2021R1A2B5B02002167, 2021M3H4A1A02056037, and \\ 2021M3F3A2A03017873) and the Basic Science Research Program through the National Research Foundation of Korea, funded by the Ministry of Science, ICT \& Future Planning (2020R1A2C2010984). \\ Author details \\ ${ }^{1}$ School of Advanced Materials Science and Engineering, Sungkyunkwan University, Suwon, Korea. ${ }^{2}$ SKKU Advanced Institute of Nanotechnology (SAINT), Sungkyunkwan University, Suwon, Korea. ${ }^{3}$ Department of Electronic Engineering, Gachon University, 1342 Seongnam-daero, Seongnam, Korea}

\section{Author contributions}

M.N. and K.H.C. mainly performed the experiments and wrote the paper. H.Y., S.C., B.J.K., S.O., J.J., C.W., N.L., S.K., and J.Y.C. supported the experiment and performed electrical measurements. S.K. and J.Y.C. managed and advised this research.

\section{Conflict of interest}

The authors declare no competing interests.

\section{Publisher's note}

Springer Nature remains neutral with regard to jurisdictional claims in published maps and institutional affiliations.

Supplementary information The online version contains supplementary material available at https://doi.org/10.1038/s41427-021-00314-y.

Received: 25 September 2020 Revised: 18 January 2021 Accepted: 20 April 2021.

Published online: 28 May 2021

\section{References}

1. Nomura, K. et al. Room-temperature fabrication of transparent flexible thinfilm transistors using amorphous oxide semiconductors. Nature $\mathbf{4 3 2}$, 488-492 (2004).

2. Kim, M. G., Kanatzidis, M. G., Facchetti, A. \& Marks, T. J. Low-temperature fabrication of high-performance metal oxide thin-film electronics via combustion processing. Nat. Mater. 10, 382-388 (2011).

3. Wang, Z., Nayak, P. K., Caraveo-Frescas, J. A. \& Alshareef, H. N. Recent developments in p-type oxide semiconductor materials and devices. Adv. Mater. 28, 3831-3892 (2016).

4. Zhao, C. et al. A. Evaporated tellurium thin films for p-type field-effect transistors and circuits. Nat. Nanotechnol. 15, 53-58 (2020).

5. Fortunato, E., Barquinha, P. \& Martins, R. Oxide semiconductor thin-film transistors: a review of recent advances. Adv. Mater. 24, 2945-2986 (2012).

6. Park, J. W., Kang, B. H. \& Kim, H. J. A Review Of Low-temperature Solutionprocessed Metal Oxide Thin-film Transistors For Flexible Electronics. Adv. Funct. Mater. 30, 1904632 (2020).

7. Martinez-Landeros, V. H. et al. Low-temperature thin film transistors based on pulsed laser deposited CdS active layers. Semicond. Sci. Technol. 34, 025008 (2019).

8. Yeom, H. I., Ko, J. B., Mun, G. \& Park, S. H. K. High mobility polycrystalline indium oxide thin-film transistors by means of plasma-enhanced atomic layer deposition. J. Mater. Chem. C. 4, 6873-6880 (2016).

9. Sirringhaus, H. 25th anniversary article: organic field-effect transistors: the path beyond amorphous silicon. Adv. Mater. 26, 1319-1335 (2014).

10. Fortunato, E., Barquinha, P. \& Martins, R. Oxide semiconductor thin-film transistors: a review of recent advances. Adv. Mater. 24, 2945-2986 (2012).

11. Powell, M. J. The physics of amorphous-silicon thin-film transistors. IEEE Trans. Electron Devices 36, 2753-2763 (1989). 
12. Fischer, A. G., Tizabi, D. J. \& Blanke, H. p-type thin-film transistors with vacuumdeposited crystallized copper-doped germanium films. IEEE electron device Lett. 4, 447-448 (1983)

13. Park, $\mathrm{H}$. et al. High-density integration of carbon nanotubes via chemical selfassembly. Nat. Nanotech. 7, 787-791 (2012).

14. Kim, S., Kim, S., Park, J., Ju, S. \& Mohammadi, S. Fully transparent pixel circuits driven by random network carbon nanotube transistor circuitry. ACS Nano 4 , 2994-2998 (2010).

15. Chen, B. et al. Highly uniform carbon nanotube field-effect transistors and medium scale integrated circuits. Nano Lett. 16, 5120-5128 (2016).

16. Cao, Q. et al. Arrays of single-walled carbon nanotubes with full surface coverage for high-performance electronics. Nat. Nanotech. 8, 180 (2013).

17. Shahrjerdi, D., Hekmatshoar, B., Mohajerzadeh, S. S., Khakifirooz, A. \& Robertson, M. High mobility poly-Ge thin-film transistors fabricated on flexible plastic substrates at temperatures below $130^{\circ} \mathrm{C}$. J. Electron. Mater. 33, 353-357 (2004).

18. Wang, $Y$. et al. Field-effect transistors made from solution-grown twodimensional tellurene. Nat. Electron 1, 228-236 (2018).

19. Zhou, G. et al. High-mobility helical tellurium field-effect transistors enabled by transfer-free, low-temperature direct growth. Adv. Mater. 30, 1803109 (2018).

20. Amani, M. et al. Solution-synthesized high-mobility tellurium nanoflakes for short-wave infrared photodetectors. ACS Nano 12, 7253-7263 (2018).

21. Wu, B., Liu, X., Yin, J. \& Lee, H. Bulk $\beta$-Te to few layered $\beta$-tellurenes: indirect to direct band-Gap transitions showing semiconducting property. Mater. Res. Express 4, 095902 (2017).

22. Yi, S., Zhu, Z., Cai, X., Jia, Y. \& Cho, J. H. The nature of bonding in bulk tellurium composed of one-dimensional helical chains. Inorg. Chem. 57, 5083-5088 (2018).

23. Von Hippel, A. Structure and conductivity in the $\mathrm{VI} b$ group of the periodic system. J. Chem. Phys. 16, 372-380 (1948).

24. Skadron, P. \& Johnson, V. A. Anisotropy and annealing behavior in extrinsic single-crystal tellurium. J. Appl. Phys. 37, 1912-1917 (1966).

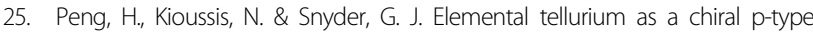
thermoelectric material. Phys. Rev. B 89, 195206 (2014).

26. Agapito, L. A., Kioussis, N., Goddard, W. A. III \& Ong, N. P. Novel family of chiralbased topological insulators: elemental tellurium under strain. Phys. Rev. Lett. 110, 176401 (2013).

27. Dutton, R. W. \& Muller, R. S. Electrical properties of tellurium thin films. Proc. IEEE 59, 1511-1517 (1971).

28. Okuyama, K., Yamamoto, H. \& Kumagai, Y. Effect of Au nucleation centers and deposition rate on crystallinity and electronic properties of evaporated Te films. J. Appl. Phys. 46, 105-111 (1975).

29. Dutton, R. W. \& Muller, R. S. Large grain tellurium thin films. Thin Solid Films 11, 229-236 (1972).
30. Qian, H. S., Yu, S. H., Gong, J. Y., Luo, L. B. \& Fei, L. F. High-quality luminescent tellurium nanowires of several nanometers in diameter and high aspect ratio synthesized by a poly (vinyl pyrrolidone)-assisted hydrothermal process. Langmuir 22, 3830-3835 (2006).

31. Zhao, A. W., Ye, C. H., Meng, G. W., Zhang, L. D. \& Ajayan, P. M. Tellurium nanowire arrays synthesized by electrochemical and electrophoretic deposition. J. Mater. Res. 18, 2318-2322 (2003).

32. Zhu, Y. J., Wang, W. W., Qi, R. J. \& Hu, X. L. 2004. Microwave-assisted synthesis of single-crystalline tellurium nanorods and nanowires in ionic liquids. Angew. Chem. Int. Ed. 43, 1410-1414 (2004).

33. Mayers, B. \& Xia, Y. One-dimensional nanostructures of trigonal tellurium with various morphologies can be synthesized using a solution-phase approach. J. Mater. Chem. 12, 1875-1881 (2002).

34. Yu, H., Gibbons, P. C. \& Buhro, W. E. Bismuth, tellurium, and bismuth telluride nanowires. J. Mater. Chem. 14, 595-602 (2004)

35. Qin, J. K. et al. Raman response and transport properties of tellurium atomic chains encapsulated in nanotubes. Nat. Electron 3, 141-147 (2020).

36. Medeiros, P. V. et al. Single-atom scale structural selectivity in Te nanowires encapsulated inside ultranarrow, single-walled carbon nanotubes. ACS Nano 11, 6178-6185 (2017).

37. Kobayashi, K. \& Yasuda, H. Structural transition of tellurium encapsulated in confined one-dimensional nanospaces depending on the diameter. Chem. Phys. Lett. 634, 60-65 (2015).

38. Nieto-Ortega, B. et al. Band-gap opening in metallic single-walled carbon nanotubes by encapsulation of an organic salt. Angew. Chem. Int. Ed. 56, 12240-12244 (2017)

39. Komsa, H. P., Senga, R., Suenaga, K. \& Krasheninnikov, A. V. Structural distortions and charge density waves in iodine chains encapsulated inside carbon nanotubes. Nano Lett. 17, 3694-3700 (2017).

40. Pine, A. S. \& Dresselhaus, G. Raman spectra and lattice dynamics of tellurium. Phys. Rev. B 4, 356 (1971).

41. Isomäki, H. M. \& von Boehm, J. Optical absorption of tellurium. Phys. Scr. $\mathbf{2 5}$ 801 (1982).

42. Swan, R., Ray, A. K. \& Hogarth, C. A. Optical absorption in thin tellurium films. Phys. Status Solidi A 127, 555-560 (1991).

43. Sangwan, V. K. et al. Fundamental performance limits of carbon nanotube thin-film transistors achieved using hybrid molecular dielectrics. ACS Nano 6 7480-7488 (2012).

44. Yu, J., Kim, B. J., Park, S., Han, I. K. \& Kang, S. J. Red/green/blue selective phototransistors with a hybrid structure of quantum-dots and an oxide semiconductor. Jpn J. Appl. Phys. 57, 044001 (2018). 